\title{
Beta talasemia intermedia: características clínicas y estudio molecular. Serie de casos clínicos
}

\author{
Beta thalassemia intermedia: clinical characteristics and molecular analysis. \\ Case series
}

\author{
Bioq. Silvia Eandi Eberle ${ }^{a}$ Bioq. Carolina Pepe ${ }^{a}$, Bioq. Fernando Aguirre, Bioq. Berenice Milanesio ${ }^{a}$, Bioq. Diego \\ Fernández ${ }^{a}$, Bioq. Adrián Mansini ${ }^{a}$ Lic. Alejandro Cháveza ${ }^{a}$ Dra. Gabriela Sciuccati ${ }^{a}$, Dra. Lilian Díaz ${ }^{a}$ \\ Dra. Andrea Candása , Dra. Vanesa Avalos Gómeza , Dra. Mariana Bonduel y Dra. Aurora Feliú Torres ${ }^{a}$
}

\begin{abstract}
RESUMEN
La beta talasemia intermedia es una hemoglobinopatía de amplio espectro clínico, que surge de la presencia de una o dos mutaciones en el gen $H B B$, asociada a modificadores genéticos secundarios y/o terciarios. Analizamos las características clínicas y de laboratorio de 29 pacientes con beta talasemia intermedia, evaluados en un período de 23 años. La edad mediana fue de 10,8 años (rango: 0,34-60,4). El 100\% de los pacientes mostró anemia microcítica hipocrómica, y solo el $17,2 \%$ presentó esplenomegalia y requerimiento transfusional esporádico. El análisis molecular de los pacientes detectó 3 con los dos genes $H B B$ afectados; 2 con un gen $H B B$ afectado y genes alfa cuadriplicados/triplicados; 23 con un gen $H B B$ afectado y genes alfa triplicados; y 1 con dos genes $H B B$ afectados y polimorfismos de genes gama. La correcta identificación de estos pacientes aseguró un adecuado consejo genético y la implementación de controles clínicos regulares.

Palabras clave: beta talasemia intermedia, modificadores genéticos, análisis molecular.
\end{abstract}

\begin{abstract}
Betathalassemiaintermediaisaquantitativehaemoglobinopathy covering a broad clinical spectrum, that results from the presence of one or two $H B B$ gene mutations associated with secondary and/or tertiary genetic modifiers. We analyze the clinical and laboratory features of 29 patients with beta thalassemia intermedia, assessed over a period of 23 years.

Median age was 10.8 years (range: 0.34-60.4). Hypochromic microcytic anemia was seen in $100 \%$ of the patients, while only $17.2 \%$ had splenomegaly and occasional transfusion requirement. The molecular analysis of patients detected: 3 with two $H B B$ affected genes; 2 with one $H B B$ affected gene and alpha quadruplicate/triplicate genes; 23 with one $H B B$ affected gene and alpha triplicate genes and 1 with two $H B B$ affected genes and polymorphisms of gamma genes. The adequate identification of these patients enables us to give
\end{abstract}

a. Servicio de Hematología y Oncología, Hospital de Pediatría "Prof. Dr. J. P. Garrahan", Buenos Aires.

Correspondencia:

Dra. Aurora Feliú Torres: afeliu@garrahan.gov.ar

Financiamiento: Ninguno.

Conflicto de intereses: Ninguno que declarar.

Recibido: 11-3-2015

Aceptado: 22-4-2015 appropriate genetic counseling and implementation of regular clinical follow up.

Key words: beta thalassemia intermedia, gene modifiers, molecular analysis.

http:/ /dx.doi.org/10.5546/aap.2015.e294

\section{INTRODUCCIÓN}

La hemoglobina es un tetrámero formado por dos cadenas tipo $\alpha$ y dos cadenas tipo no $\alpha$. En los individuos normales mayores de un año de edad, aproximadamente el $95 \%$ de la hemoglobina es hemoglobina $A\left(\alpha_{2} \beta_{2}\right)$, con menos de $3,5 \%$ de hemoglobina $A_{2}\left(\alpha_{2} \delta_{2}\right)$ y menos de $1 \%$ de hemoglobina fetal $\left(\alpha_{2} \gamma_{2}\right){ }^{1}$

Las hemoglobinopatías abarcan un grupo diverso de trastornos causados por la alteración del patrón normal de expresión de los genes de globina. Estos trastornos se caracterizan por la síntesis reducida o ausente de una o más cadenas de globina (talasemias) o por la síntesis de una hemoglobina estructuralmente anormal (hemoglobinopatías estructurales). Se estima que el $7 \%$ de la población mundial es portadora de alguno de estos desórdenes. ${ }^{2} \mathrm{La} \beta$ talasemia (BT) es causada por mutaciones en el gen $H B B$, que se localiza en el cromosoma 11. Las mutaciones pueden suprimir completamente (mutaciones $\beta^{0}$ ) o disminuir (mutaciones $\beta^{+} \mathrm{y}$ $\beta^{++}$) la producción de cadenas $\beta$ globina, lo que resulta en un desequilibrio en la síntesis de cadenas de globina $\alpha / \beta$. La magnitud de este es la determinante principal del fenotipo de la enfermedad, que abarca desde los individuos asintomáticos (BT menor o portador) hasta los que dependen de transfusiones regulares para vivir (BT mayor). Entre ambos extremos, se encuentran los pacientes con $\beta$ talasemia intermedia (BTI), en los cuales las manifestaciones clínicas son el resultado de la interacción de modificadores genéticos y ambientales. ${ }^{3}$

El objetivo de este trabajo es presentar las características clínicas y de laboratorio de los 
pacientes con BTI diagnosticadas en el Servicio de Hematología y Oncología del Hospital de Pediatría "Prof. Dr. Juan P. Garrahan".

\section{OBSERVACIÓN}

Se realizó el estudio retrospectivo, descriptivo, longitudinal de 29 individuos (12 varones y 17 mujeres) pertenecientes a 19 familias no relacionadas, con diagnóstico de BTI, que consultaron entre marzo de 1991 y marzo de 2014 (Tabla 1).

El diagnóstico se basó en el estudio de patología eritrocitaria, al propósito y su grupo familiar, que incluyó la historia clínica, el hemograma completo (Sysmex XS 800i, Sysmex Corporation, Kobe, Japan), el examen del frotis de sangre periférica, el recuento reticulocitario, la electroforesis de hemoglobina semiautomatizada en gel de agarosa a $\mathrm{pH}$ alcalino y la electroforesis capilar (Sebia, Lisses, Évry, France), la cuantificación de hemoglobina $\mathrm{A}_{2}$ por cromatografía de intercambio aniónico (Helena), la cuantificación de hemoglobina fetal por desnaturalización alcalina, la prueba de resistencia osmótica eritrocitaria, el metabolismo del hierro y la prueba de Brewer. ${ }^{4}$

Los estudios moleculares fueron realizados en ADN aislado de leucocitos de sangre periférica por el método de precipitación salina. Las mutaciones puntuales en el gen $H B B$ más frecuentes en la población argentina (IVSI-6, IVSI-110, IVSI-1, IVSII-745, CD39, IVSII-1 y CD6 (-A)) fueron analizadas por reacción en cadena de la polimerasa (polymerase chain reaction; $\mathrm{PCR}$, por sus siglas en inglés) específica de alelo utilizando primers descritos previamente..$^{5,6}$ Las mutaciones puntuales o pequeñas inserciones-deleciones no buscadas en la pesquisa inicial por PCR específica de alelo fueron estudiadas por PCR-Secuenciación utilizando primers descritos previamente por Roldán y col., y un secuenciador automático ABI PRISM 3130 (Applied Biosystems). ${ }^{7}$

Las deleciones más frecuentes en el cluster $\alpha$ globina $\left(-\alpha^{3.7}\right.$ y $\left.-\alpha^{4.2}\right)$ fueron analizadas por GAP-PCR utilizando los primers y la estrategia descrita previamente por Chong y col. con algunas modificaciones. ${ }^{8,9}$ La presencia de genes $\alpha$ triplicados-cuadruplicados anti-3,7 fue analizada por PCR según ha sido descrito previamente y los genes $\alpha$ triplicados/cuadriplicados generados por mecanismos diferentes al que da origen al alelo anti-3,7 fueron identificados por amplificación de sondas dependiente de ligandos múltiples (Multiplex Ligation-dependent Probe Amplification;
MLPA, por sus siglas en inglés), utilizando el kit comercial Salsa MLPA P140B HBA (MRCHolland) ${ }^{10}$

La variante genética $H B G 2:$ c. $-211 \mathrm{C}>\mathrm{T}$ $(-158 \mathrm{C}>\mathrm{T}, \mathrm{rs7482144)}$ fue analizada por PCRRFLP (Restriction Fragment Length Polymorphism; polimorfismos en la longitud de los fragmentos de restricción), utilizando la enzima de restricción XmnI. ${ }^{11}$ Las variantes genéticas HBS1LMYB rs9399137 T>C y BCL11A rs11886868 T>C y rs1427407 G > T se analizaron por PCRSecuenciación en el Ospedale Regionale Microcitemie, Cagliari, Italia.

En el período mencionado, se estudiaron 5802 individuos. Los síndromes talasémicos representaron el $25,9 \%$ de los diagnósticos efectuados, de los cuales un $2 \%$ fueron BTI. La mediana de edad de los 29 individuos fue de 10,8 años (rango: 0,34-60,4).

Todos los pacientes consultaron por anemia microcítica hipocrómica y, a excepción de la \#29, presentaron hemoglobina $\mathrm{A}_{2}$ aumentada con concentraciones variables de hemoglobina fetal y metabolismo del hierro normal. El 17,2\% de los pacientes (del \#1 al \#5) presentaron, además de la anemia microcítica hipocrómica, requerimiento transfusional esporádico y esplenomegalia. El análisis molecular del gen $H B B$ en 3 de los 5 pacientes (del \#1 al \#3) evidenció ambos alelos afectados, mientras que los dos pacientes restantes (el \#4 y el \#5) presentaron un solo alelo $\beta$ afectado en presencia de genes $\alpha$ cuadriplicados (paciente \#5) y de genes $\alpha$ triplicados/cuadriplicados (paciente \#4). E1 79,3\% de los pacientes (23) presentaron un único alelo $\beta$ afectado asociado a genes $\alpha$ triplicados.

La paciente \#29 presentó anemia microcítica moderada con $0,9 \%$ de hemoglobina $\mathrm{A}_{2}$ y $88 \%$ de hemoglobina fetal. El estudio molecular del gen $H B B$ identificó la mutación $\beta^{0}$ (CD 39) en homocigosis y el cluster $\alpha$ normal. Ante la ausencia de esplenomegalia, anomalías óseas y requerimiento transfusional, se planteó la búsqueda de modificadores genéticos secundarios fuera del cluster de $\beta$ globina y asociados a la producción aumentada de hemoglobina fetal.

Se estudió un marcador genético localizado en la región intergénica $H B S 1 L-M Y B$ y dos marcadores genéticos localizados en el gen $B C L A 11 A$, y se observó, en dicha paciente, la presencia heterocigota y homocigota, respectivamente, de los genotipos asociados a síntesis aumentada de cadenas $\gamma$ y de hemoglobina fetal. 


\section{DISCUSIÓN}

La BT presenta fenotipos clínicos y hematológicos de gravedad variable. Así, se reconocen individuos con BT menor, que presentan solo anemia microcítica hipocrómica leve sin manifestaciones clínicas y, en el otro extremo, se en-

TABLA 1. Características clínicas y de laboratorio

\begin{tabular}{|c|c|c|c|c|c|c|c|c|c|c|}
\hline $\begin{array}{l}\text { Paciente } \\
\text { (flia.) }\end{array}$ & $\begin{array}{c}\text { Edad } \\
\text { (años) }\end{array}$ & $\begin{array}{c}\text { Clínica } \\
\text { E. físico }\end{array}$ & $\begin{array}{l}\text { Hemoglobina } \\
\text { (g/dl) }\end{array}$ & $\begin{array}{c}\text { VCM } \\
\text { (fl) }\end{array}$ & $\begin{array}{l}\text { Hemoglobina } \\
\text { fetal }(\%)\end{array}$ & $\begin{array}{c}\text { Hemoglobina } \\
\mathrm{A}_{2}(\%)\end{array}$ & $\begin{array}{c}\text { a Genotipo } \\
\text { HBB } \\
\end{array}$ & $\begin{array}{l}\text { Genotipo } \\
\text { grupo } \alpha\end{array}$ & $\begin{array}{c}\text { HBG2 } \\
158 \mathrm{C}>\mathrm{T}\end{array}$ & Otros \\
\hline \# 1 (1) & 3 & $\begin{array}{l}\text { MH RTGR } \\
\text { lenomegalia }\end{array}$ & 5,8 & 73,8 & 42 & 3,8 & I-110/I-6 & $\alpha \alpha / \alpha \alpha$ & Ausente & NA \\
\hline \# 2 (2) & ${ }^{3,3}$ & $\begin{array}{l}\text { MH RTGR } \\
\text { blenomegalia }\end{array}$ & 8,1 & 72,0 & 33,7 & 4,1 & $\mathrm{I}(-3) / \mathrm{I}(-3)$ & $\alpha \alpha / \alpha \alpha$ & Ausente & NA \\
\hline \# 3 (3) & ${ }^{7,1}$ e & $\begin{array}{l}\text { MH RTGR } \\
\text { lenomegalia }\end{array}$ & 9,6 & 59,2 & 13,5 & 5,7 & $\mathrm{I}-2 /-101$ & $\alpha \alpha / \alpha \alpha$ & Ausente & NA \\
\hline$\# 4$ (4) & $\begin{array}{l}3,6 \\
\mathrm{e}\end{array}$ & $\begin{array}{l}\text { MH RTGR } \\
\text { lenomegalia }\end{array}$ & 7,5 & 64,6 & 18 & 3,9 & $\beta^{\mathrm{A}} / \mathrm{CD} 39$ & $\alpha \alpha \alpha / \alpha \alpha \alpha \alpha$ & Ausente & NA \\
\hline \# 5 (5) & $\begin{array}{r}10,4 \\
\text { es }\end{array}$ & $\begin{array}{l}\text { MH RTGR } \\
\text { lenomegalia }\end{array}$ & $a$ & 67,5 & 30 & 4,2 & $\beta^{\mathrm{A}} / \mathrm{CD} 39$ & $\alpha \alpha / \alpha \alpha \alpha \alpha$ & Ausente & NA \\
\hline \# 6 (6) & 4 & $\mathrm{AMH}$ & 8,7 & 59,7 & 1,0 & 3,8 & $\beta^{\mathrm{A}} / \mathrm{II}-1$ & $\alpha \alpha / \alpha \alpha \alpha$ & Ausente & NA \\
\hline \# 7 (6) & 1,9 & $\mathrm{AMH}$ & 9,3 & 56,3 & 2,2 & 4,2 & $\beta^{\mathrm{A}} / \mathrm{II}-1$ & $\alpha \alpha / \alpha \alpha \alpha$ & Ausente & NA \\
\hline \# 8 (7) & 29,6 & $\mathrm{AMH}$ & 9,6 & 64,8 & 3,7 & 5,3 & $\beta^{\mathrm{A}} / \mathrm{CD} 39$ & $\alpha \alpha / \alpha \alpha \alpha$ & Ausente & NA \\
\hline \# 9 (7) & 60,4 & $\mathrm{AMH}$ & 8,6 & 72,9 & 5,9 & 6,0 & $\beta^{\mathrm{A}} / \mathrm{CD} 39$ & $\alpha \alpha / \alpha \alpha \alpha$ & Ausente & NA \\
\hline \# 10 (8) & 44,3 & $\mathrm{AMH}$ & 10,9 & 62,7 & 2,4 & 5 & $\beta^{\mathrm{A}} / \mathrm{CD} 39$ & $\alpha \alpha / \alpha \alpha \alpha$ & Hetero- & NA \\
\hline \# 11 (8) & 11,7 & $\mathrm{AMH}$ & 10,6 & 58,6 & 2,4 & 4,2 & $\beta^{\mathrm{A}} / \mathrm{CD} 39$ & $\alpha \alpha / \alpha \alpha \alpha$ & Hetero- & NA \\
\hline \# 12 (8) & 26,7 & $\mathrm{AMH}$ & 8,6 & 57,1 & 3,0 & 4,1 & $\beta^{\mathrm{A}} / \mathrm{CD} 39$ & $\alpha \alpha / \alpha \alpha \alpha$ & Hetero- & NA \\
\hline \# 13 (8) & 1,2 & $\mathrm{AMH}$ & 10,3 & 46,1 & 2,9 & 5,3 & $\beta^{\mathrm{A}} / \mathrm{CD} 39$ & $\alpha \alpha / \alpha \alpha \alpha$ & Hetero- & NA \\
\hline \# 14 (9) & 45,9 & $\mathrm{AMH}$ & 11,4 & 73,1 & 13,4 & 3,6 & $\beta^{\mathrm{A}} / \mathrm{CD} 39$ & $\alpha \alpha / \alpha \alpha \alpha$ & Ausente & NA \\
\hline \# 15 (10) & 10,4 & $\mathrm{AMH}$ & 9,7 & 52,3 & 0,4 & 5,3 & $\beta^{\mathrm{A}} / \mathrm{CD} 39$ & $\alpha \alpha / \alpha \alpha \alpha$ & Hetero- & NA \\
\hline \# 16 (10) & 34,1 & $\mathrm{AMH}$ & 11,1 & 52,9 & 0,9 & 5,1 & $\beta^{\mathrm{A}} / \mathrm{CD} 39$ & $\alpha \alpha / \alpha \alpha \alpha$ & Ausente & NA \\
\hline \# 17 (11) & 27,8 & $\mathrm{AMH}$ & 12,0 & 57,2 & 0,5 & 4,2 & $\beta^{\mathrm{A}} / \mathrm{CD} 39$ & $\alpha \alpha / \alpha \alpha \alpha$ & Ausente & NA \\
\hline \# 18 (12) & 37,5 & $\mathrm{AMH}$ & 10,0 & 61,9 & 5,4 & 5,1 & $\beta^{\mathrm{A}} / \mathrm{CD} 6-\mathrm{A}$ & $\alpha \alpha / \alpha \alpha \alpha$ & Hetero- & NA \\
\hline \# 19 (13) & 1 & $\mathrm{AMH}$ & 9,8 & 58,5 & 11,4 & 3,6 & $\beta^{\mathrm{A}} / \mathrm{I}-110$ & $\alpha \alpha / \alpha \alpha \alpha$ & NA & NA \\
\hline \# 20 (13) & 35,4 & $\mathrm{AMH}$ & 11,8 & 63,1 & 0,6 & 5,0 & $\beta^{\mathrm{A}} / \mathrm{I}-110$ & $\alpha \alpha / \alpha \alpha \alpha$ & NA & NA \\
\hline \# 21 (14) & 19 & $\mathrm{AMH}$ & 9,9 & 69,0 & 0,9 & 5,5 & $\beta^{\mathrm{A}} / \mathrm{I}-110$ & $\alpha \alpha / \alpha \alpha \alpha$ & Hetero- & NA \\
\hline \# 22 (14) & 25,7 & $\mathrm{AMH}$ & 11,4 & 67,8 & 0,9 & 4,2 & $\beta^{\mathrm{A}} / \mathrm{I}-110$ & $\alpha \alpha / \alpha \alpha \alpha$ & Hetero- & NA \\
\hline \# 23 (15) & 0,3 & $\mathrm{AMH}$ & 7,4 & 59,5 & 13,6 & 4,5 & $\beta^{\mathrm{A}} / \mathrm{II}-745$ & $\alpha \alpha / \alpha \alpha \alpha$ & Ausente & NA \\
\hline \# 24 (16) & 27,4 & $\mathrm{AMH}$ & 11,4 & 66,0 & 2,7 & 4,3 & $\beta^{\mathrm{A}} / \mathrm{I}-110$ & $\alpha \alpha / \alpha \alpha \alpha$ & NA & NA \\
\hline \# 25 (17) & 13,6 & $\mathrm{AMH}$ & 9,8 & 61,4 & 0,25 & 5,0 & $\beta^{\mathrm{A}} / \mathrm{c} .44 \mathrm{delT}$ & $\alpha \alpha / \alpha \alpha \alpha$ & Hetero- & NA \\
\hline \# 26 (17) & 10,8 & $\mathrm{AMH}$ & 10,7 & 60,6 & 1,7 & 5,7 & $\beta^{\mathrm{A}} / \mathrm{c} .44 \mathrm{delT}$ & $\alpha \alpha / \alpha \alpha \alpha$ & Hetero- & NA \\
\hline \# 27 (17) & 2,9 & $\mathrm{AMH}$ & 10,3 & 54,5 & 14,4 & 4,4 & $\beta^{\mathrm{A}} / \mathrm{c} .44 \mathrm{delT}$ & $\alpha \alpha / \alpha \alpha \alpha$ & Homo- & NA \\
\hline \# 28 (18) & 2,1 & $\mathrm{AMH}$ & 8,7 & 54,4 & 0,9 & 6,1 & $\beta^{\mathrm{A}} / \mathrm{I}-1$ & $\alpha \alpha / \alpha \alpha \alpha$ & NA & NA \\
\hline \# 29 (19) & 1,5 & $\mathrm{AMH}$ & 9,7 & 69,9 & 88,4 & 0,9 & CD39/CD39 & $\alpha \alpha / \alpha \alpha$ & $\begin{array}{r}\text { Ausente } \\
\text { T } \\
\text { B }\end{array}$ & $\begin{array}{c}\text { HBS1L- } \\
\text { MYB rs9399137 } \\
\text { T>C:T } / C ; \text { BCL11A } \\
\text { rs11886868 } \\
\text { T>C:C/C } \\
\text { BCL11A rs1427407 } \\
\text { G>T:T } / \mathrm{T}\end{array}$ \\
\hline
\end{tabular}

$\beta^{\mathrm{A}}$ : alelo normal; NA: no analizado; Hetero-: heterocigota; Homo-: homocigota; AMH: anemia microcítica hipocrómica; RTGR: requerimiento transfusional de glóbulos rojos esporádico; VCM: volumen corpuscular medio. 
cuentran los pacientes con BT mayor, con anemia grave desde el primer año de vida y la necesidad de transfusiones regulares y tratamiento quelante para sobrevivir. ${ }^{3}$ En 1955, Sturgeon acuñó el término BTI para incluir a aquellos pacientes en los cuales las manifestaciones clínicas y hematológicas eran de gravedad intermedia entre la BT menor y la mayor. Dicha variabilidad resulta de la combinación de mutaciones en el o los genes de $\beta$ globina (modificadores primarios), asociada a otras mutaciones (modificadores secundarios) que aumentan o disminuyen el desequilibrio en la producción de las diferentes cadenas de globina, lo que altera el grado de eritropoyesis ineficaz y sus consecuencias (anemia, visceromegalias, sobrecarga de hierro). ${ }^{12}$

Las mutaciones de los genes $\beta$ (modificadores primarios) se clasifican en $\beta^{0}, \beta^{+}$y $\beta^{++}$dependiendo del grado de síntesis residual de globina $\beta$ y, consecuentemente, de la posibilidad de síntesis de hemoglobina A.

Entre los modificadores genéticos secundarios que aumentan el desequilibrio $\alpha / \beta$, se destaca la presencia de genes $\alpha$ triplicados o cuadruplicados; mientras que la co-herencia de $\alpha$ talasemia y de las variantes genéticas que aumentan la producción de hemoglobina fetal disminuyen tal desequilibrio.

En nuestra experiencia de 23 años, los síndromes $\beta$ talasémicos representaron el 25,9\% de los diagnósticos realizados; las BTI fueron el $2 \%$ de ellos. En la población estudiada, es necesario resaltar las diferencias clínicas entre los pacientes que presentaron dos mutaciones en los genes de $\beta$ globina de aquellos en los cuales sólo se identificó una mutación en dicho gen, asociada a la presencia de mayor número de genes $\alpha$ o a modificadores de la producción de hemoglobina fetal. Este último grupo de pacientes fue derivado por presentar anemia microcítica hipocrómica sin manifestaciones clínicas excepto una leve palidez o para evaluación hematológica por el antecedente familiar de $\beta$ talasemia. Las marcadas diferencias en la morfología eritrocitaria y los índices hematimétricos observadas entre estos pacientes y sus familiares permitieron sospechar la presencia de modificadores secundarios (Figura 1. $A$ y $1 . B)$.

La BTI, actualmente incluida dentro del grupo de las talasemias no dependientes de transfusiones, no está exenta de complicaciones serias. Los estudios realizados por Taher y col., demuestran que la morbilidad, mayor a la previamente reconocida, aumenta con el avance de la edad. La sobrecarga de hierro, subvalorada por la ferritina sérica, se desarrolla aun en pacientes no transfundidos, lo que exige la implementación de métodos diagnósticos no invasivos, tales como la resonancia magnética nuclear hepática y cardíaca. El daño óseo, los focos de eritropoyesis extramedular, las visceromegalias, las úlceras en los miembros inferiores y la predisposición a trombosis son algunas de las complicaciones observadas a lo largo de la vida de estos pacientes. Todo esto exige la identificación temprana y correcta de

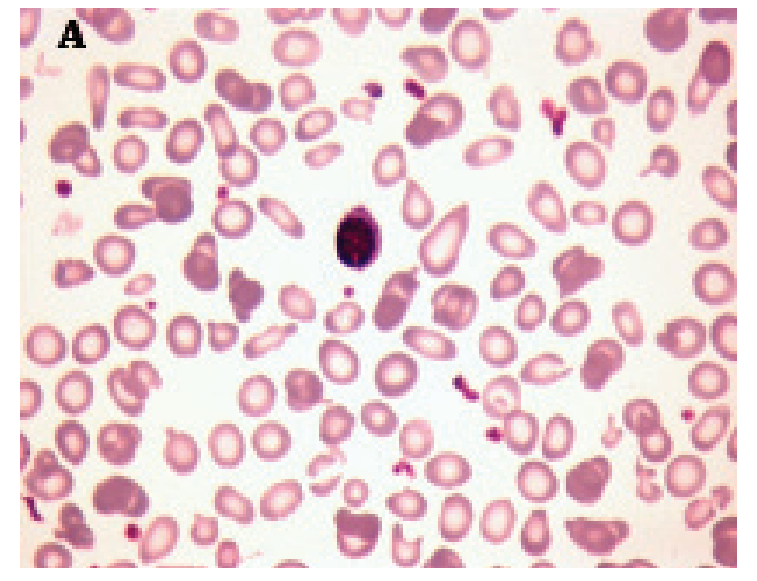

A. Paciente con beta talasemia intermedia.

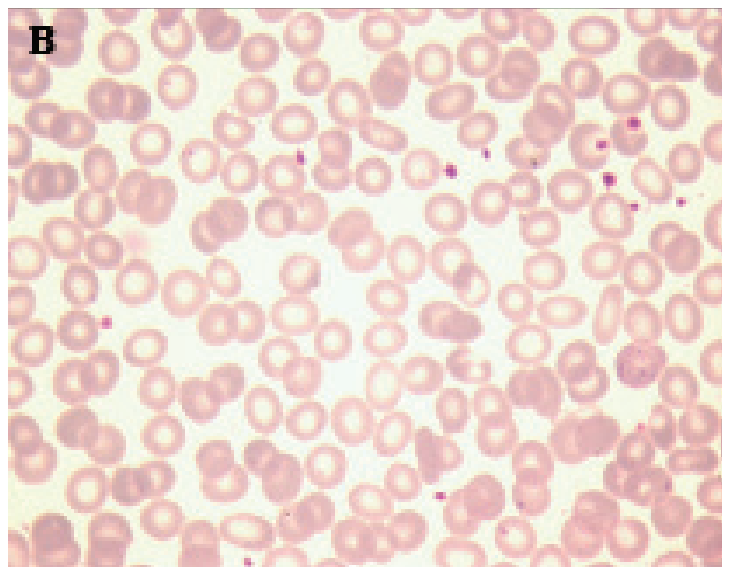

B. Paciente con beta talasemia heterocigota. 
esta patología para asegurar el consejo genético e implementar los controles clínicos regulares con la correspondiente intervención terapéutica. ${ }^{13}$

\section{REFERENCIAS}

1. Bain BJ. Chapter 1, Haemoglobin and the genetics of haemoglobin synthesis. En: Haemoglobinopathy diagnosis. $2^{\text {nd }}$ ed. Massachusetts: Blackwell Publishing; 2006. Págs.1-25.

2. Weatherall DJ. The challenge of haemoglobinopathies in resource-poor countries. Br J Haematol 2011;154(6):736-44.

3. Rund D, Rachmilewitz E. Beta-thalassemia. N Engl J Med 2005;353(11):1135-46.

4. Roper D, Layton M. Investigation of the hereditary haemolyticanaemias:membraneand enzymeabnormalities. En: Lewis SM, Bain BJ, Bates I, eds. Dacie and Lewis Practical Haematology. $10^{\text {th }}$ ed. Philadelphia: Churchill Livingston; 2006. Págs.205-37.

5. Fortina P, Dotti G, Conant R, Monokian G, et al. Detection of the most common mutations causing beta-thalassemia in Mediterraneans using a multiplex amplification refractory mutation system (MARMS). PCR Methods Appl 1992;2(2):163-6.

6. Old JM, Varawalla NY, Weatherall DJ. Rapid detection and prenatal diagnosis of beta-thalassaemia:studies in Indian and
Cypriot populations in UK. Lancet 1990;336(8719):834-7.

7. Roldán A, Gutiérrez M, Cygler A, Bonduel M, et al. Molecular characterization of beta-thalassemia genes in an Argentine population. Am J Hematol 1997;54(3):179-82.

8. Chong SS, Boehm CD, Cutting GR, Higgs DR. Simplified multiplex-PCR diagnosis of common southeast asian deletional determinants of alpha-thalassemia. Clin Chem 2000;46(10):1692-5.

9. Pepe C, Eberle SE, Chaves A, Milanesio B, et al. A new $\beta(0)$ frameshift mutation, HBB: c.44delT (p.Leu14ArgfsX5), identified in an Argentinean family associated with secondary genetic modifiers of $\beta$-thalassemia. Hemoglobin 2014;38(6):444-6.

10. Wang W, Ma ES, Chan AY, Prior J, et al. Single-tube multiplex-PCR screen for anti-3.7 and anti-4.2 alpha-globin gene triplications. Clin Chem 2003;49(10):1679-82.

11. Lanclos KD, Oner C, Dimovski AJ, Gu YC, et al. Sequence variations in the $5^{\prime}$ flanking and IVS-II regions of the G gamma- and A gamma-globin genes of beta Schromosomes with five different haplotypes. Blood 1991;77(11):2488-96.

12. Sturgeon $P$, Itano HA, Bergren WR. Genetic and biochemical studies of intermediate types of Cooley's anaemia. $\mathrm{Br} J$ Haematol 1955;1(3):264-77.

13. Taher A, Isma'eel H, Cappellini MD. Thalassemia intermedia: revisited. Blood Cells Mol Dis 2006;37(1):12-20. 